\title{
Studying Chemistry at the University of Zurich in the Sixties and Seventies of the Last Century: A Personal View
}

\author{
Martin Karpf*
}

Abstract: The author describes his diploma and $\mathrm{PhD}$ studies at the University of Zurich and the significant influence Prof. André Dreiding had on his development as a chemist and a person.

Keywords: Institute of Organic Chemistry UZH

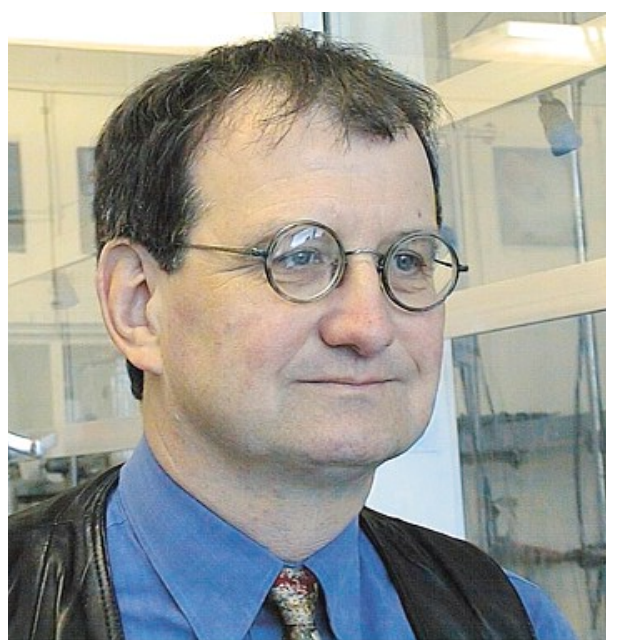

Martin Karpf

\section{Studying Chemistry at the University of Zürich in the 1960s}

The arena: the aula of the University of Zürich crowded by several hundred palavering first-year medical, natural sciences and chemistry students, nervous but enthusiastic. The event: the first lecture of general chemistry in the winter semester on a sunny October morning in 1965. A man wearing a white turtleneck pullover, sitting below a huge flickering white Eidophor television projection screen, slowly raising his right arm, a starting pistol in his hand. He fires the gun without warning. The sharp retort and a billowing white cloud instantaneously stopped all discussion and paved the way for Prof. André Dreiding's first philosophical diversion about the intellectual and per-

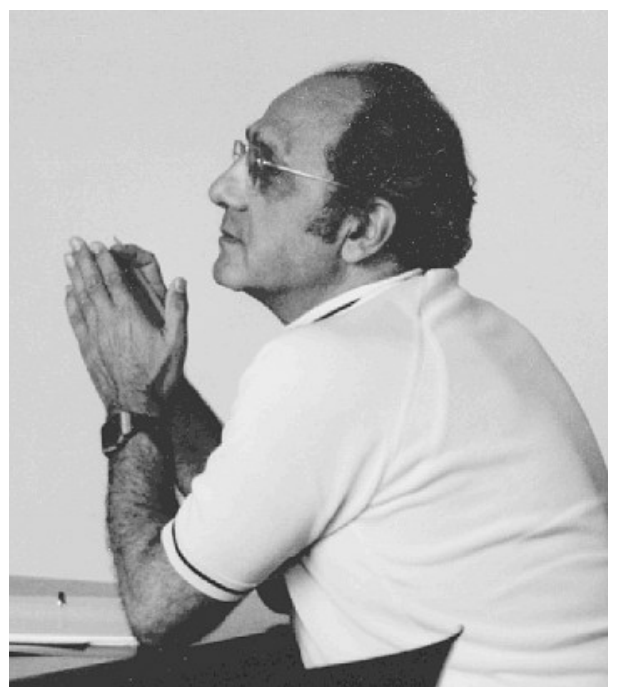

André Dreiding sonal prerequisites a young person needs to study natural sciences when everybody had been expecting a chemistry lecture instead.

This early encounter with far-reaching intellectual thoughts about science induced my later decision to join André Dreiding's group even after enduring all the issues associated with the sufferings of pre-diploma studies such as the 'Hausprüfungen' in inorganic, organic and physical chemistry, the endless practical courses and the Monday tutorials in organic chemistry embracing each time at least three chapters of Cram, Hammond, ${ }^{[1]}$ a stressful 'survival of the fittest' event personally supervised by Prof. Hans Schmid and his assistants.

\section{Embarking on Diploma and PhD Thesis in André Dreiding's Research Group}

Although it was well known amongst undergraduates that joining Prof. Dreiding's group might eventually be accompanied by an extended duration of studies, the abovementioned initial attraction was further increased by his profound and thoughtful courses in stereochemistry. Indeed, after the first discussion about potential topics to work on for a diploma or $\mathrm{PhD}$ thesis, the deeply impressed students left his office with the feeling of being becoming the rising stars of future science. Looking back, the moot point is: was all that extensive time spent in discussing algorithms for the generation of unequivocal names for molecules, ${ }^{[2]}$ contemplating chirality, absolute asymmetric syntheses and the classification 
of stereochemical terms and definitions worth it? No, if one thinks in straightforward terms of efficiency, productivity and yield as being a generally accepted measure for the effectiveness of a competitive research group but yes, if one regards the chemistry department as still being part of the philosophical faculty of the university.

The examination of current chemical literature in the famous problem seminars was one of the methods for sharpening critical minds. These were prepared in turn by advanced students and post-docs by transforming spectroscopic and structural information of recently published results into a puzzle to be discussed and solved by the attending colleagues. Irresolvable problems frequently left questions which often led to the initiation of a diploma thesis such as on Ugi's postulated existence of penta-coordinated carbon atoms in transition states. ${ }^{[3]}$ In group meetings, André Dreiding's critical outbreaks of skepticism sometimes provoked an uproar as in e.g. his questioning the enantiomeric ratios of different fractions collected from the chromatographic purification of nonracemic mixtures on a silica gel column. How could he ponder about a change in the ratio using an achiral support? This penetrating question finally led to the previously generally neglected but now commonly accepted fact ${ }^{[4]}$ that enantiomeric differentiation of nonracemic mixtures in an achiral environment is an important far-reaching phenomenon and a caveat for the accurate determination of enantiomer ratios and their interpretation.

The stimulating atmosphere was an active culture medium for new ideas, inventions and discoveries such as the first synthesis of the betalain type red dye of beetroot, ${ }^{[5]}$ the first triaziridine ${ }^{[6]}$ finally secured after many years of passionate research, the $\alpha$-alkynone cyclization ${ }^{[7]}$ representing the thermal cyclization of $\alpha$-aetylenic ketones to 2-cyclopentenones, a discovery hypothesis based upon a trace by-product of a totally different thermal reaction. This cyclization - although proceeding at $600^{\circ} \mathrm{C}$ in a quartz tube - provided high yields of product and permitted the later synthesis of various polyquinane natural products including the first propellane structure found in nature, modhephene. ${ }^{[8]}$

Writing publications with André Dreiding, however, was an exhaustive endeavor comparable to climbing Mount Everest. Only solid proof that a reasoning in the twelfth version was exactly the same or at least extremely similar to one in the fifth version could, after an additional few months of maturation, hopefully persuade André Dreiding to consider the paper to be ready for submission. His hypercritical mind never tolerated a word, a sentence, an argument in a publication to appear without profound and thoughtful discussions that now and then were held on weekends at his marvelous home in Herrliberg. These meetings were interrupted only when being spoiled and treated to snacks by his charming wife Norma or by André's sudden proposal to watch the Sunday evening thriller on TV in order to relax.

\section{Leaving the Institute Prepared for Future Challenges}

Whatever position the members of the group eventually reached after obtaining their degrees, they universally succeeded, whether in industry, academia or even in banking based on André Dreiding's firm conviction that in studying chemistry, regardless of the specific topic worked on during the thesis, one would wind up with a keen mind prepared to succeed in any other business. In his belief, being trained as a chemist meant knowing 'the game' whereas ignoring facts and wishful thinking left one invariably failing in the next step.

\section{Acknowledgements}

The careful reading of the manuscript by Paul Spurr is gratefully acknowledged.

Received: January 29, 2008
[1] D. J. Cram, G. S. Hammond, 'Organic Chemistry', 2nd ed., McGraw Hill, New York, 1964, $846 \mathrm{pp}$

[2] A. Hess, A. S. Dreiding, H. R. Haegi, 'Classification of mobile molecules by category theory', in 'Symmetries and Properties of Non-Rigid Molecules: A Comprehensive Survey', Eds. J. Maruani, J. Serre, Proceedings of an International Symposium, Paris, France, 1-7 July 1982, Studies in Physical and Theoretical Chemistry 1983, 23, 39-5; P. Floersheim, K. Wirth, M. K. Huber, D. Pazis, F. Siegerist, H. R. Haegi, A. S. Dreiding, 'From mobile molecules to their symmetry groups: A computerimplemented method', Studies in Physical and Theoretical Chemistry 1983, 23, 59-80.

[3] T. Vergnani, M. Karpf, L. Hoesch, A.S Dreiding, 'Has the existence of intermediates with pentacoordinated carbon atoms in aliphatic SN2 reactions been proven?', Helv. Chim. Acta 1975, 58, 2524.

[4] W. L. Tsai, K. Hermann, E. Hug, B. Rohde, A. S. Dreiding, 'Enantiomerdifferentiation induced by an enantiomeric excess during chromatography with achiral phases', Helv. Chim. Acta 1985 68, 2238. See also: V. A. Soloshonok, 'Remarkable amplification of the selfdisproportionation of enantiomers on achiral-phase chromatography columns', Angew. Chem. In. Ed. 2006, 45, 766.

[5] K. Hermann, A. S. Dreiding, 'Total synthesis of betalaines', Helv. Chim. Acto 1977, 60, 673 .

[6] C. Leuenberger, L. Hoesch, A.S. Dreiding, 'A triaziridine', J. Chem. Soc. Chem. Comm. 1980, 24, 1197.

[7] M. Karpf, A.S. Dreiding, 'Thermal cyclizations of $\alpha$-alkinones zu 2-cyclopentenones', Helv. Chim. Acta 1979, 62, 852

[8] M. Karpf, A.S. Dreiding, 'Application of the a-alkynone cyclization: Synthesis of rac-modhephene', Tetrahedron. Lett. 1980, $21,4569$. 